\title{
Advances in the analysis of short span railway bridges for high-speed lines
}

\author{
P. Museros ${ }^{\text {a,* }}$, M.L. Romero ${ }^{\mathrm{a}}$, A. Poy ${ }^{\mathrm{a}}$, E. Alarcón ${ }^{\mathrm{b}}$ \\ ${ }^{a}$ Departamento de Tecnología, Universitat Jaume I, 12071 Castellón, Spain

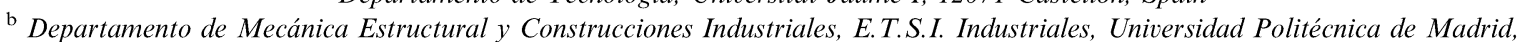 \\ ClJosé Gutiérrez Abascal 2, 28006 Madrid, Spain
}

\begin{abstract}
The physical model based on moving constant loads is widely used for the analysis of railway bridges. Nevertheless, the moving loads model is not well suited for the study of short bridges $(L \leqslant 20-25 \mathrm{~m})$ since the results it produces (displacements and accelerations) are much greater than those obtained from more sophisticated ones. In this paper two factors are analysed which are believed to have an influence in the dynamic behaviour of short bridges. These two factors are not accounted for by the moving loads model and are the following: the distribution of the loads due to the presence of the sleepers and ballast layer, and the train-bridge interaction. In order to decide on their influence several numerical simulations have been performed. The results are presented and discussed herein.
\end{abstract}

Keywords: Dynamics of railway bridges; Moving loads; Train-bridge interaction; Resonance speed; Short bridges; High-speed lines; Distribution through ballast

\section{Introduction}

The dynamic behaviour of railway bridges has been a subject of research for many scientists and engineers since the first accidents occurred in metal bridges during the past century. Some of the most remarkable works on this subject are those by Stokes [19], Bresse [4], Willis [22], Bleich [3], Inglis [13], Timoshenko [21] and Frýba $[10,11]$, among others.

From these works it can be observed that the physical model most frequently used for the dynamic analysis of railway bridges is the so-called moving loads model. This model does not take into account the inertial effects of the train masses, and therefore the train is modelled as a series of concentrated, constant-valued loads travelling at speed $V$ (see Fig. 1). For this model closed-form solutions of the equations of motion can be obtained in a very simple manner $[2,10,18,21]$.

The response computed with the moving loads model at non-resonance speeds is in good agreement with experiments as shown in $[9,12]$. Nevertheless, at resonance speeds bridge displacements and accelerations can be significantly magnified leading to dangerous situations.

The temporary closure of the TGV line from Paris to Lyon is a good example of the problems that may arise in high-speed lines. In that case, excessively high accelerations were detected in several bridges that lead to ballast liquefaction and, as a consequence, danger of derailment [14,17]. In November 1995, the European Rail Research Institute (ERRI), located in the Netherlands, decided to create a committee of experts (ERRI D-214) in charge of the study of such problems. In one of the works by the ERRI D-214 committee [7] it can be observed that for the short spans the vertical accelerations of the deck predicted by the moving loads model 


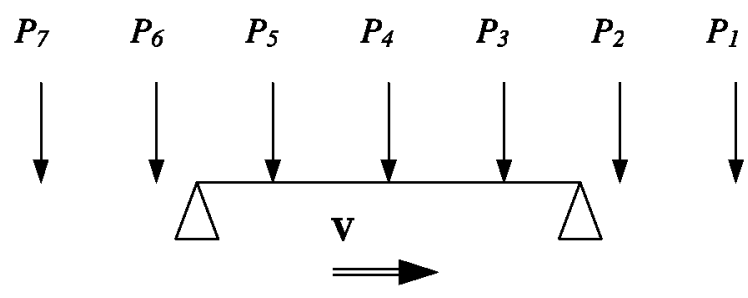

Fig. 1. Moving loads model.

reach very high values. These values are much greater than the limit acceleration related to the appearance of ballast liquefaction, which is about $7 \mathrm{~m} / \mathrm{s}^{2} \approx 0.7 \mathrm{~g}$, i.e. $70 \%$ of the acceleration of gravity (see Fig. 2).

Unfortunately, as the ERRI D-214 points out in its Final Report [9], there is a lack of experimental data recorded at resonance speeds that precludes the possibility of comparing predicted values with real ones. In Spain tests at resonance speeds have been conducted in several bridges [1] but no comparison with theoretical values has been performed; moreover, the numerical simulations presented by the authors of [1] were pointing to adjust mechanical properties of the model in order to fit with experimental results, rather than comparing predicted and measured values.

At non-resonance speeds, bridge response predicted by more sophisticated models including train-bridge interaction is very similar to the one obtained from moving loads models. Conversely, train-bridge interaction significantly reduces displacements and accelera- tions at resonance, which could be of great interest from an economic point of view. As shown in [8], the effects of train-bridge interaction are indeed noticeable for short bridges, which are most likely to undergo excessive resonant vibration. Even if an extensive comparison with experiments is still required for resonance situations, simplified procedures enabling civil engineers to account for train-bridge interaction in short bridges are most desirable, since values predicted by moving loads models (as the ones in Fig. 2) are too high and have never been observed in real tests.

In this paper the influence of two factors not accounted for by the moving loads model is analysed: the first one is the distribution of the loads through the sleepers and ballast layer; the second one is the trainbridge interaction. Other factors that are believed to play an important role in the dynamics of short bridges are the following:

- Values of damping during the passage of the train greater than the ones computed from the logarithmic decrement of the free oscillation.

- Boundary restrictions exerted by the rail in the transitions over the abutments.

- Vibration of the ballast layer.

Finally, another factor that is central to the dynamics of railway bridges is the excitation due to track irregularities and wheel flats. As it is well known, the peak response of high-speed railway bridges is obtained in

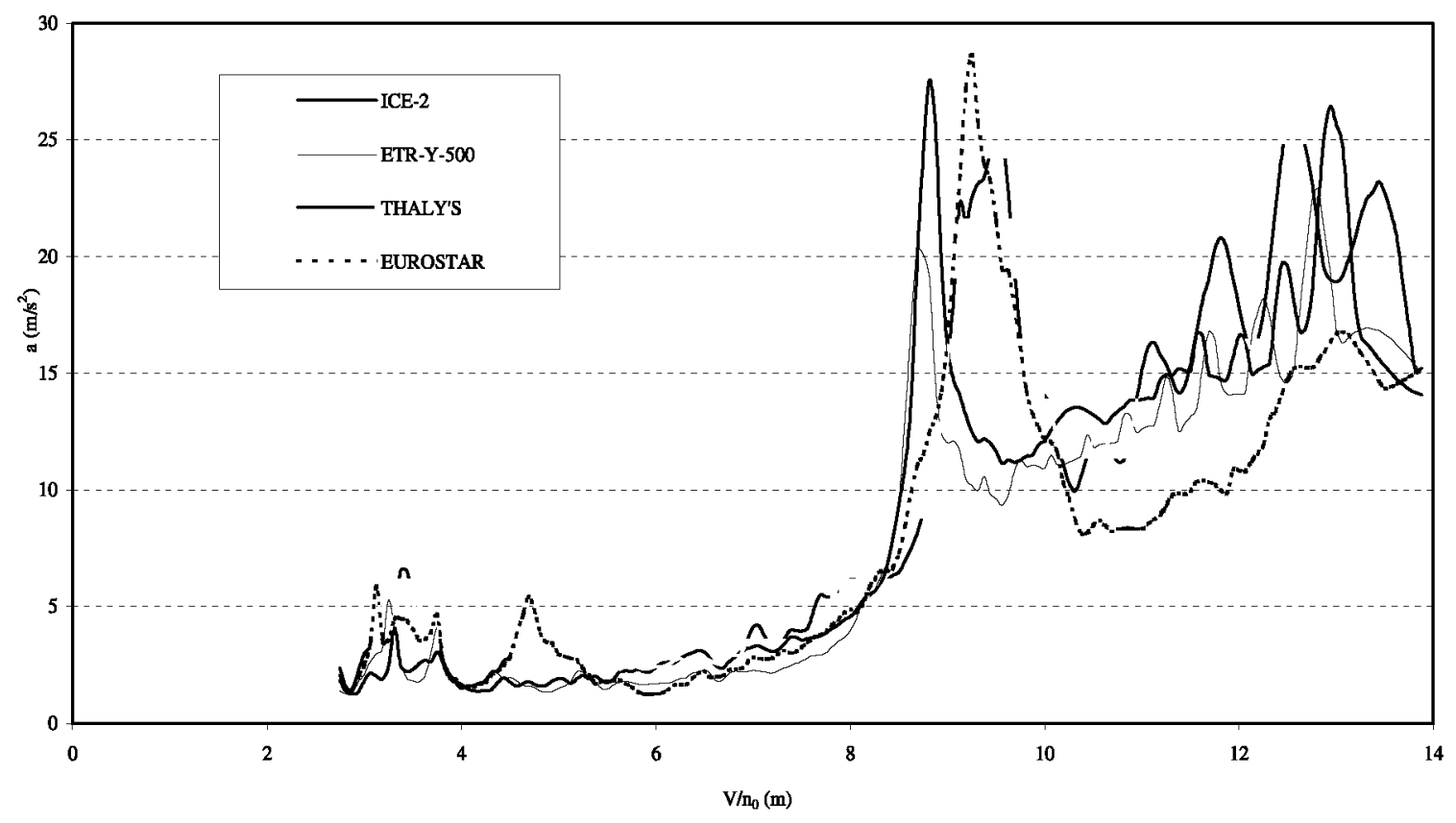

Fig. 2. Maximum accelerations in a bridge of $\operatorname{span} L=10 \mathrm{~m}, n_{0}=8 \mathrm{~Hz}$. Mass per unit length $=10,000 \mathrm{~kg} / \mathrm{m}$. 
correspondence of the resonance speeds in most cases. Although the effects of track irregularities and wheel flats are of considerable importance for non-resonance speeds, they have not been taken into account in this study, which is focussed mainly on the behaviour of bridges at resonance. The influence of such factors in resonance situations is presently being investigated by other authors [6].

\section{Numerical modelling}

In order to compute the deflections and accelerations three computer programs have been developed. The mathematical bases of the different models can be reviewed in the works by several authors [2,10,18,21]. The two first programs are based on the physical model of a simply supported beam crossed by a series of concentrated or distributed loads, respectively. According to this model, the dynamic behaviour of the bridge is governed by the well-known partial differential equation:

$m \frac{\partial^{2} y(x, t)}{\partial t^{2}}+E I \frac{\partial^{4} y(x, t)}{\partial x^{4}}=q(x, t)$

where $y(x, t)$ is the vertical deflection of the beam at point $x$ and time $t ; m$, the constant mass of the beam per unit length; $E$, the modulus of elasticity; $I$, the constant moment of inertia of the cross-section of the beam; and $q(x, t)$, the load acting per unit length at point $x$ and time $t$. In both cases, for concentrated (i.e., represented by means of Dirac delta functions) as well as distributed loads, a closed-form solution for Eq. (1) can be obtained and no numerical integration is required.

The third program, on the contrary, is able to analyse continuous girders crossed by a train of sprung and semi-sprung masses, and therefore can be used to treat the train-bridge interaction problem. This program uses two-dimensional Bernoulli beam elements in order to represent the behaviour of the bridge, and a set of concentrated masses, linear springs and dampers to account for the characteristics of the train (Fig. 3).

The integration is carried out by means of a modified Newmark- $\beta$ method [20]. The mass, damping and stiffness matrices of the whole system are updated at every time step, and the displacements of the axles, as well as

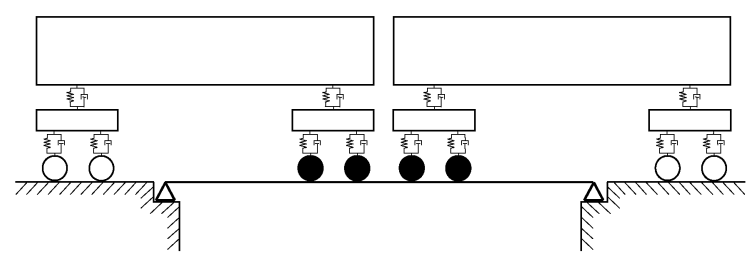

Fig. 3. Train-bridge model. the train-bridge interaction forces, are interpolated by means of the usual cubic Hermitian polynomials. The chosen time step is at least one one hundredth the time required by any of the train axles to cross the bridge's span. If this condition is satisfied the behaviour of the system can be considered linear within the step and no iterative procedures are required; using a longer time step has proved sometimes inaccurate for the computation of the maximum displacements and accelerations. In order to ensure the desired accuracy, two additional restrictions are considered in the selection of the time step: it has to be shorter than 0.05 times the period of the third mode of the bridge, and also shorter than 0.05 times the period of the higher mode of the train. The results obtained with the third program have been checked against those presented by the ERRI D-214 committee in the Train-Bridge Interaction report [8] and the ones available in Ref. [23].

\section{Distribution of the loads through the sleepers and ballast layer}

In order to analyse the effects of the distribution of the loads beneath the sleepers and ballast layer (Fig. 4), the similarity formulae proposed by ERRI [7,16] are taken as a departure point. These formulae are valid for the responses computed by the concentrated and distributed loads models and are as follows:

$\Phi=\frac{f\left(L, \zeta, m, n_{0}, \frac{V}{n_{0}}\right)}{f_{\mathrm{LM} 71}}=\frac{f^{\prime}\left(L, \zeta, m^{\prime}, n_{0}^{\prime}, \frac{V^{\prime}}{n_{0}^{\prime}}\right)}{f_{\mathrm{LM} 71}^{\prime}}$

$a_{\max }\left(L, \zeta, m, n_{0}, \frac{V}{n_{0}}\right)=\frac{m^{\prime}}{m} a_{\max }^{\prime}\left(L, \zeta, m^{\prime}, n_{0}^{\prime}, \frac{V^{\prime}}{n_{0}^{\prime}}\right)$

In these formulae $\Phi$ is the impact coefficient, i.e., the relation between dynamic and static deflections at midspan; $f$ and $f^{\prime}$ are the maximum vertical deflections at mid-span of two bridges of the same length (henceforth

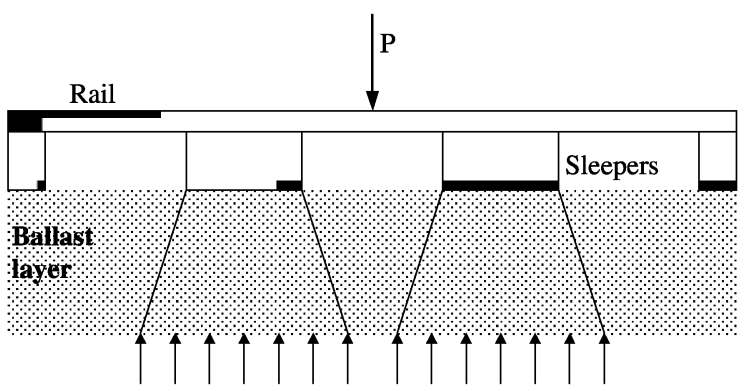

Fig. 4. Distribution of the axle loads through the sleepers and ballast layer. 
called first and second bridges); $f_{\mathrm{LM} 71}$ and $f_{\mathrm{LM} 71}^{\prime}$ are the static deflections at mid-span of the two bridges due to the Load Model $71 ; a_{\max }$ and $a_{\max }^{\prime}$ are the maximum vertical accelerations at mid-span; $L$ is the length or span of the bridges and $\zeta$ is the damping ratio. The variables that define the dynamic behaviour of the bridges are $m$ and $m^{\prime}$, which are the mass of the bridges per unit length, as well as $n_{0}$ and $n_{0}^{\prime}$, which are the fundamental frequencies. Finally, $V$ is the speed of the train passing over the first bridge (mass $m$, frequency $n_{0}$ ) and $V^{\prime}$ is the speed of the train passing over the second bridge (mass $m^{\prime}$, frequency $n_{0}^{\prime}$ ).

The Load Model 71 is a static load pattern proposed by the Eurocode-1 [5] for the design of railway bridges. For computing the static deflection $f_{\text {LM71 }}$ a classification factor $\alpha=1.21$ has been used. This is permitted by the Eurocode-1 and is also imposed by the new Spanish Code of Actions in Railway Bridges [15].

It should be emphasised that the similarity formulae are valid provided that the wavelength $\lambda$ has the same value for both bridges:

$\lambda=\frac{V}{n_{0}}=\frac{V^{\prime}}{n_{0}^{\prime}}=\lambda^{\prime}$

As can be observed from $\mathrm{Eq}$. (2a), the value of the impact coefficient computed with the moving loads model is equal for all the bridges of the same length and damping ratio provided that the wavelengths are the same.

Now the reductions of the displacements $(R)$ and accelerations $\left(R^{\prime}\right)$ due to the load distribution through the sleepers and ballast are defined as follows:

$R=\frac{\Phi_{\mathrm{c}}-\Phi_{\mathrm{d}}}{\Phi_{\mathrm{c}}} \times 100$

$R^{\prime}=\frac{a_{\max , \mathrm{c}}-a_{\max , \mathrm{d}}}{a_{\max , \mathrm{c}}} \times 100$

In Eqs. (4a) and (4b), subscripts "c" and "d" stand for "concentrated loads" and "distributed loads" respectively. The analysis with the distributed loads model has been performed assuming that each of the axle loads acts uniformly over a length of $1 \mathrm{~m}$ (approximately). This value is found by averaging the ones that would be obtained if the scheme of distribution suggested by Eurocode- 1 were applied to the two extreme situations, i.e. the axle load acting directly over one of the sleepers and the axle lying in the middle point between two adjacent sleepers. A 25 -cm-thick ballast layer has been considered for these computations.

Since the value of the impact coefficient is the same for all the bridges of the same length and damping, the same holds for the reduction of displacements $R$. Besides, considering Eq. (4b), the same property is found to be valid for the reduction of the accelerations $R^{\prime}$.
Therefore the reductions defined in Eqs. (4a) and (4b) have been evaluated for nine reference bridges of spans ranging from 4 to $15 \mathrm{~m}$ with a damping ratio $\zeta=0.01$. Realistic values of the damping ratio are usually greater for the shortest bridges, where the energy dissipated by the continuous rail and ballast layer is of greater importance. Nevertheless, a constant value of $1 \%$ has been selected so as to suppress the influence of damping in the reductions $R$ and $R^{\prime}$, which therefore depend solely on the value of the wavelength. The results, as stated before, are valid for any bridge having a span length equal to the length of any of the reference bridges. Figs. 5 and 6 show the maximum accelerations predicted by the

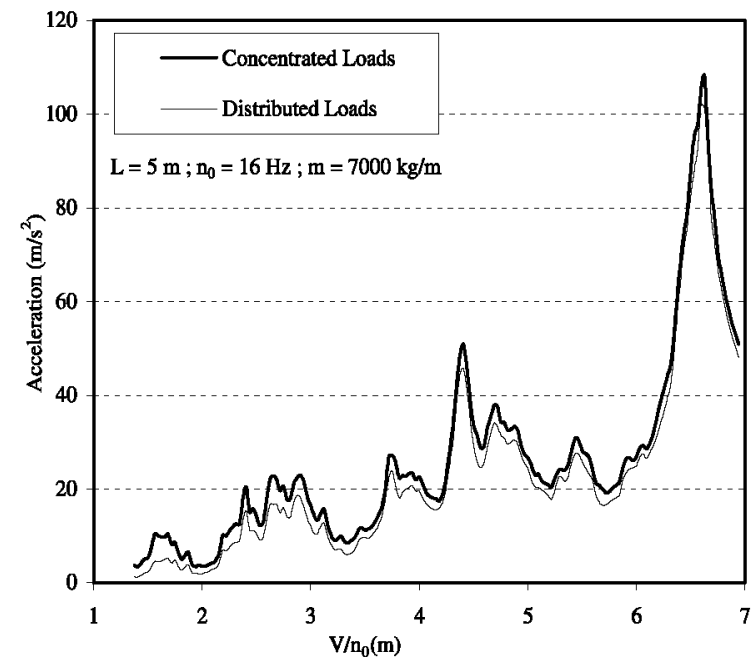

Fig. 5. Maximum accelerations in a bridge of $\operatorname{span} L=5 \mathrm{~m}$. Five European high-speed trains considered.

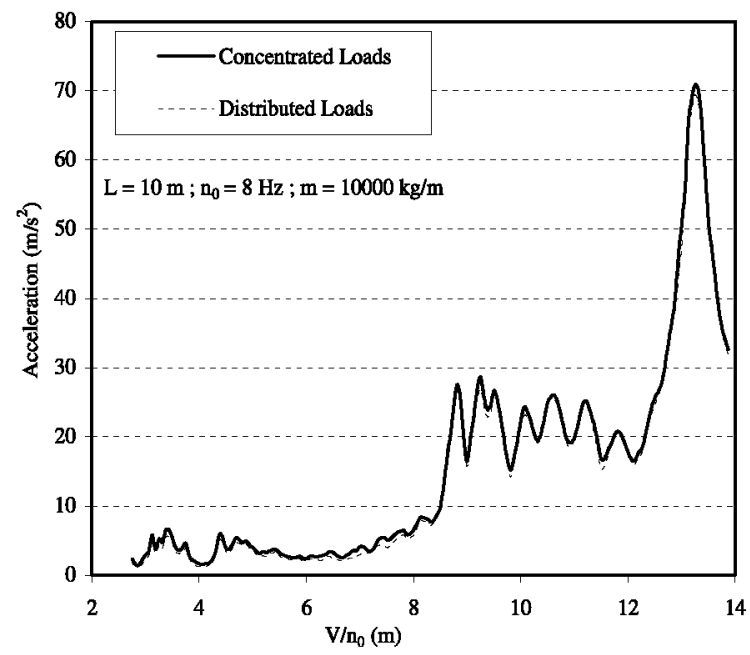

Fig. 6. Maximum accelerations in a bridge of $\operatorname{span} L=10 \mathrm{~m}$ Five European high-speed trains considered. 
concentrated and distributed loads models for the reference bridges of span length 5 and $10 \mathrm{~m}$. The results are plotted as a function of the wavelength $\lambda=V / n_{0}$. Five European high-speed trains have been considered in the analysis, including the Spanish TALGO, the French THALY'S, the German ICE-2, the Italian ETR-Y and the EUROSTAR.

As can be observed from Figs. 5 and 6, while the reductions are negligible for the $10 \mathrm{~m}$ bridges, they should not be disregarded in the $5 \mathrm{~m}$ ones (especially for the low speeds). In general, it is found that the shorter the value of the wavelength, the greater the reduction of the accelerations. Conversely, for the longer wavelengths (i.e., for bridges with a lower fundamental frequency, or traversed by trains travelling at higher speeds) the reductions decrease monotonically.

This conclusion is shown in Fig. 7, where the reduction $R^{\prime}$ is presented as a function of the wavelength for the bridges of 6,7 and $8 \mathrm{~m}$ of span length. The reduction $R^{\prime}$ appears as a very irregular function, characterised by sharp variations for small increments of $\lambda$. Nevertheless, a lower bound for the reduction can be proposed (as shown in Fig. 7) which represents a safe choice for the engineer in charge of the dynamic analysis of any simply supported bridge.

However, if the reduction $R^{\prime}$ is evaluated only for the resonance wavelengths (i.e., those related to a resonance phenomenon, as $\lambda=4.4$ or $6.6 \mathrm{~m}$ in Fig. 5) it is found that the values do not correspond to any of the peaks above of below the lower bound in Fig. 7. Indeed, the values are somewhat greater than the lower bound in most cases. This is shown in Fig. 8, where the reduction $R^{\prime}$ has been evaluated for all the resonance wavelengths that can be found in the bridges of 4,5,7 and $9 \mathrm{~m}$ of span length. Bridges of 6 and $8 \mathrm{~m}$ are not included for

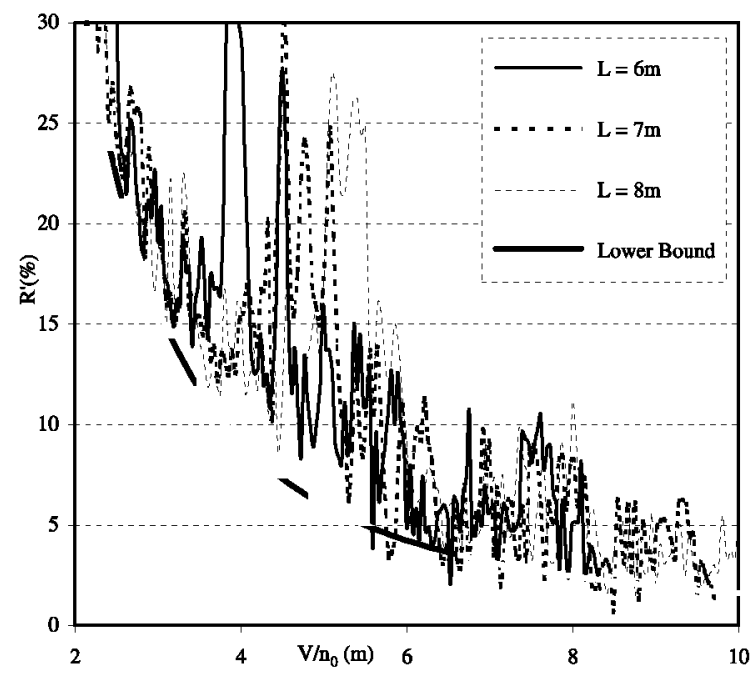

Fig. 7. Reduction of the accelerations $\left(R^{\prime}\right)$ as a function of the wavelength.

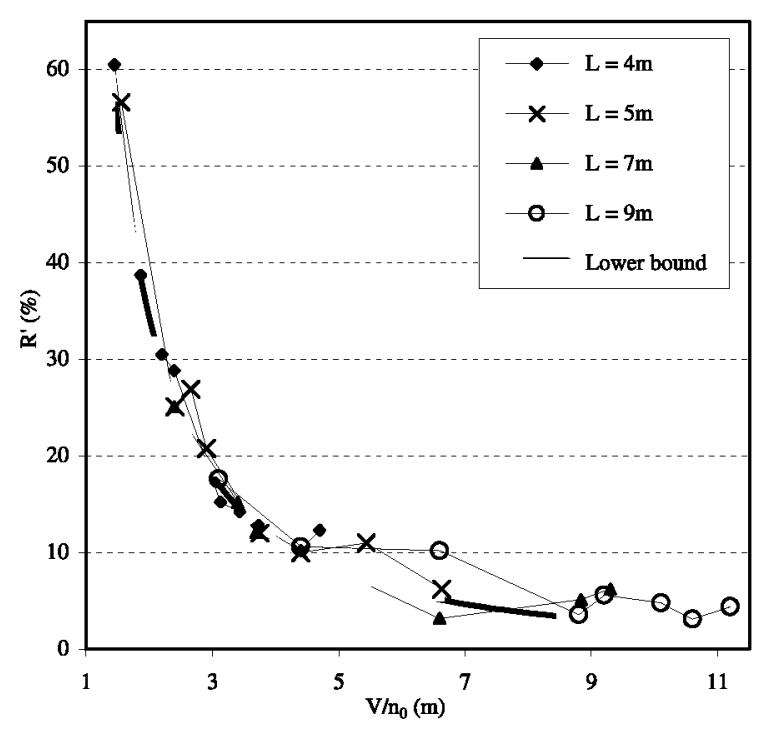

Fig. 8. Reduction of the accelerations $\left(R^{\prime}\right)$ computed for the resonance wavelengths.

the sake of clarity, and bridges longer than $9 \mathrm{~m}$ show negligible reductions for the usual range of speeds. As can be seen, a lower bound is also included that is slightly different from the one in Fig. 7. The analytical expressions for both limits are

$R^{\prime}=127.35 \lambda^{-1.9}$

for the lower bound in Fig. 7, and

$R^{\prime}=104.43 \lambda^{-1.6}$

for the one in Fig. 8. Eq. (6) is a good approximation for the reduction of accelerations, especially for the values $\lambda<4-5 \mathrm{~m}$. There are some points in Fig. 8 that lie below the lower bound, but this is of little importance since the differences are small (a more conservative expression for Eq. (6) could also be adopted). Finally, in Fig. 9 both lower bounds for $R^{\prime}$ (Eqs. (5) and (6)) are represented in logarithmic scale.

In this paper attention has been focussed on the accelerations since they are of major importance for short bridges, but similar developments can be done in a straightforward manner in order to obtain an approximation for the reduction of the impact coefficients.

\section{Train-bridge interaction}

\subsection{Introduction}

Train-bridge interaction is a phenomenon that takes place when the bridge oscillations or the rail-surface roughness excite the motion of the vehicle sprung masses. As a result, the value of the axle forces becomes time 


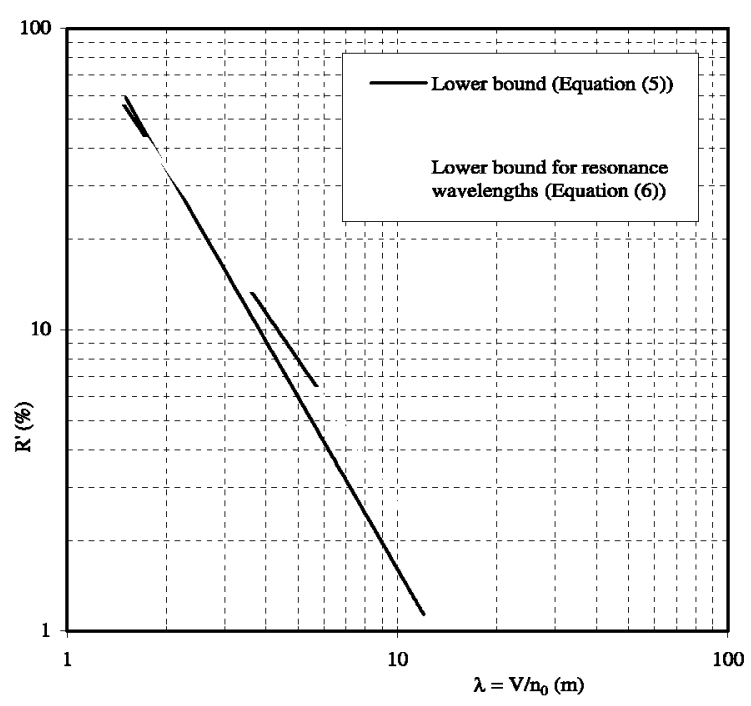

Fig. 9. Lower bounds of the reduction of the accelerations.

dependent and, therefore, it is no longer equal to the static axle load. In this paper the train-bridge interaction is investigated without considering the second of the aforementioned factors; this implies, as stated before, that solely the bridge vertical vibrations cause the motion of the sprung masses.

The D-214 committee of the ERRI showed that train-bridge interaction has a considerable influence in the dynamic behaviour of bridges having span lengths shorter than $15-20 \mathrm{~m}$ [8]. This fact was also confirmed by the authors in previous works [16], where reductions of the displacements and accelerations about $25 \%$ were found when comparing the moving loads and the interaction models. Moreover, it can be shown that the maximum reductions of the bridge response take place at the resonance speeds (or wavelengths), thus making the moving loads model excessively conservative for the design of short, simply supported bridges. In Fig. 10 the impact coefficients at the resonance wavelength $\lambda=$ $8.8 \mathrm{~m}$ for three bridges of $L=10 \mathrm{~m}$ can be seen. All the calculations in Section 4 have been performed considering the ICE-2 as the only excitation.

In principle, evaluating the reduction of the response due to the train-bridge interaction is not a simple matter. As shown in Fig. 10, the main difficulty stems from the fact that, unlike the reduction due to the load distribution through sleepers and ballast, the train-bridge interaction effects are not the same for all bridges of the same length. Therefore, a complete dynamic analysis in the time domain is required that most of the commercial finite element codes are not able to perform.

Considering that the length and damping ratio of the bridge will be kept to a constant value $(10 \mathrm{~m}$ and $1 \%$, respectively), the reductions of the displacements and accelerations can be defined as follows:

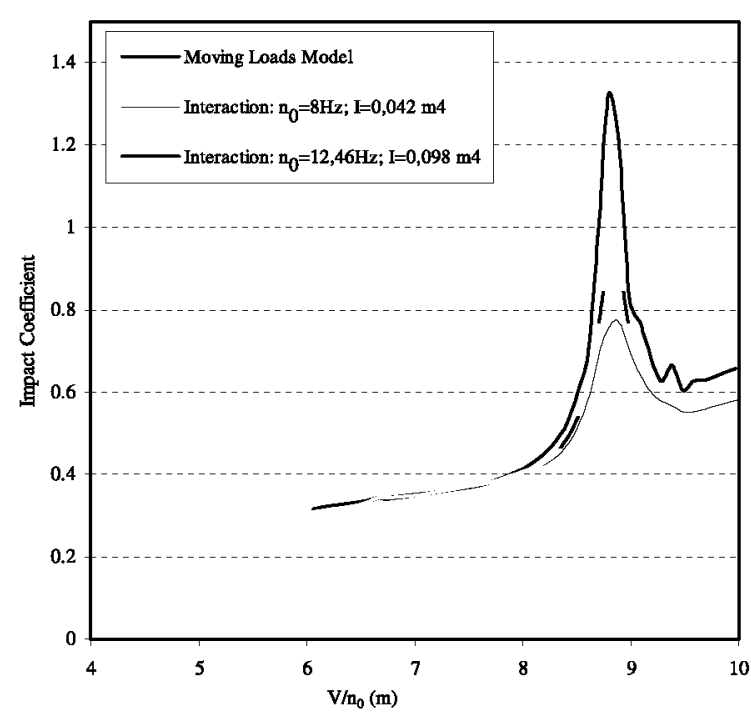

Fig. 10. Impact coefficients for three bridges of $\operatorname{span} L=10 \mathrm{~m}$.

$R\left(\lambda, n_{0}, I\right)=\frac{\Phi_{\mathrm{c}}(\lambda)-\Phi_{\mathrm{i}}\left(\lambda, n_{0}, I\right)}{\Phi_{\mathrm{c}}(\lambda)} \times 100$

$R^{\prime}\left(\lambda, n_{0}, I\right)=\frac{a_{\mathrm{c}}\left(\lambda, n_{0}, I\right)-a_{\mathrm{i}}\left(\lambda, n_{0}, I\right)}{a_{\mathrm{c}}\left(\lambda, n_{0}, I\right)} \times 100$

In Eqs. (7a) and (7b), $\Phi_{\mathrm{c}}$ and $a_{\mathrm{c}}$ are the impact coefficient and maximum acceleration computed with the moving loads model, while, $\Phi_{\mathrm{i}}$ and $a_{\mathrm{i}}$ are the ones computed taking into account the train-bridge interaction; $\lambda$ and $n_{0}$ are the usual wavelength and the natural frequency of the bridge, and $I$ is the moment of inertia of the crosssection of the beam.

From Eqs. (7a) and (7b) it can be seen that the reductions $R$ and $R^{\prime}$ depend, for a given wavelength, on the fundamental frequency of the bridge as well as on the bridge static stiffness (i.e., on the moment of inertia $I$, provided the material is given). In Ref. [16] it was shown by the authors that this was true for the reduction of the impact coefficients. In order to investigate the dependence of $R$ and $R^{\prime}$ on such variables, a parametric study has been conducted in which the behaviour of several bridges of $10 \mathrm{~m}$ of span length has been studied. Five different values of the fundamental frequency, ranging from the lower to the upper limits recommended by Eurocode-1, have been selected: $n_{01}=8 \mathrm{~Hz}$ (lower limit), $n_{02}=10.23 \mathrm{~Hz}, n_{03}=12.46 \mathrm{~Hz}, n_{04}=14.7 \mathrm{~Hz}$ and $n_{05}=16.93 \mathrm{~Hz}$ (upper limit). As can be seen, the five values of frequency are equally spaced.

Then, for every value $n_{0 i}$, five bridges with different moments of inertia have been selected. Young's modulus has been taken equal to $36 \mathrm{GPa}$, and the values of the mass per unit length and moment of inertia have been computed on the basis of two realistic requirements. 
First, the static deflection $\delta$ of the bridge due to its own weight and the Load Model 71 [5] acting simultaneously must lie between the following values: $500 \leqslant L / \delta \leqslant 3000$. Since the deflection due to self-weight is included, these limit values have been taken broader than the usual ones in the design of railway bridges, but this is a desirable feature for a parametric analysis as the one presented in this section. A classification factor $\alpha=1.21$ has been applied to the Load Model 71, as imposed by the Spanish Code of Actions. Second, the mass of the bridge per unit length must be (approx.) greater than $3000 \mathrm{~kg}$ / $\mathrm{m}$, a value which is found in some light metal bridges, and smaller than $20,000 \mathrm{~kg} / \mathrm{m}$, which is considered an upper limit for simply supported bridges of length $L=10 \mathrm{~m}$.

This makes a total amount of 25 bridges analysed, each of them for 85 values of speed ranging from 100 to $400 \mathrm{~km} / \mathrm{h}$ (i.e., from 28 to $112 \mathrm{~m} / \mathrm{s}$, with a step of $1 \mathrm{~m} / \mathrm{s}$ ). The mechanical properties of the bridges are shown in Table 1. Bridges are referenced by means of an abbreviation $B_{i j}$, where subscript $i$ indicates the value of the natural frequency and subscript $j$ indicates the value of the moment of inertia (or mass, since both are related to the frequency, as it is well known). Following this notation, bridge B21 has a natural frequency $n_{02}$ and the lowest value of inertia of the second column of the table, and bridge $\mathrm{B} 45$ has natural frequency $n_{04}$ and the highest value of inertia of the fourth column of the table.

\subsection{Approximation of the impact coefficient and maxi- mum acceleration}

Fig. 11 shows the reduction $R$ of the impact coefficients for bridges B11, B12 and B15 as a function of the wavelength. In this figure it can be observed that the reductions are nearly proportional to each other. Similarly, in Fig. 12 the reduction $R^{\prime}$ of the maximum acceleration for bridges B31, B33 and B35 is presented. Again, it seems that any of the curves in this figure can be obtained from any other multiplying by an appropriate factor. Thus, taking bridge B11 as the reference bridge, an approximation for the reductions is given by

$$
\begin{aligned}
& R\left(\lambda, n_{0}, I\right) \cong \gamma\left(n_{0}, I\right) R_{\mathrm{ref}}(\lambda) \\
& R^{\prime}\left(\lambda, n_{0}, I\right) \cong \gamma^{\prime}\left(n_{0}, I\right) R_{\mathrm{ref}}^{\prime}(\lambda)
\end{aligned}
$$

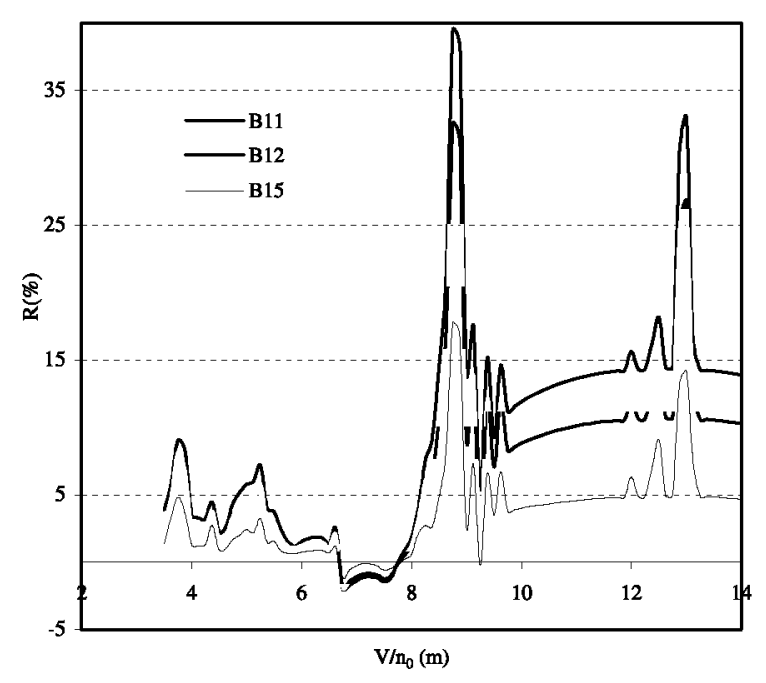

Fig. 11. Reduction of the impact coefficients for bridges B11, $\mathrm{B} 12$ and $\mathrm{B} 15$.

Table 1

\begin{tabular}{|c|c|c|c|c|c|c|c|c|c|}
\hline \multicolumn{2}{|c|}{$n_{01}=8 \mathrm{~Hz}$} & \multicolumn{2}{|c|}{$n_{02}=10.23 \mathrm{~Hz}$} & \multicolumn{2}{|c|}{$n_{03}=12.46 \mathrm{~Hz}$} & \multicolumn{2}{|c|}{$n_{04}=14.7 \mathrm{~Hz}$} & \multicolumn{2}{|c|}{$n_{05}=16.93 \mathrm{~Hz}$} \\
\hline B11 & $\begin{array}{l}L / \delta=500 \\
m=5836 \\
I=0.042045\end{array}$ & $\mathrm{~B} 21$ & $\begin{array}{l}L / \delta=500 \\
m=3165 \\
I=0.037308\end{array}$ & B31 & $\begin{array}{l}L / \delta=700 \\
m=2957 \\
I=0.051713\end{array}$ & B41 & $\begin{array}{l}L / \delta=1000 \\
m=3052 \\
I=0.074215\end{array}$ & B51 & $\begin{array}{l}L / \delta=1300 \\
m=2980 \\
I=0.096147\end{array}$ \\
\hline B12 & $\begin{array}{l}L / \delta=637.5 \\
m=8175 \\
I=0.058899\end{array}$ & $\mathrm{~B} 22$ & $\begin{array}{l}L / \delta=812.5 \\
m=5784 \\
I=0.068175\end{array}$ & B32 & $\begin{array}{l}L / \delta=1175 \\
m=5591 \\
I=0.097787\end{array}$ & B42 & $\begin{array}{l}L / \delta=1500 \\
m=5006 \\
I=0.121720\end{array}$ & B52 & $\begin{array}{l}L / \delta=1725 \\
m=4182 \\
I=0.134938\end{array}$ \\
\hline B13 & $\begin{array}{l}L / \delta=775 \\
m=11026 \\
I=0.079443\end{array}$ & $\mathrm{~B} 23$ & $\begin{array}{l}L / \delta=1125 \\
m=9148 \\
I=0.107824\end{array}$ & B33 & $\begin{array}{l}L / \delta=1650 \\
m=8988 \\
I=0.157207\end{array}$ & B43 & $\begin{array}{l}L / \delta=2000 \\
m=7362 \\
I=0.179014\end{array}$ & B53 & $\begin{array}{l}L / \delta=2150 \\
m=5532 \\
I=0.178479\end{array}$ \\
\hline B 14 & $\begin{array}{l}L / \delta=912.5 \\
m=14579 \\
I=0.105041\end{array}$ & B24 & $\begin{array}{l}L / \delta=1437.5 \\
m=13627 \\
I=0.160623\end{array}$ & B34 & $\begin{array}{l}L / \delta=2125 \\
m=13536 \\
I=0.236756\end{array}$ & B44 & $\begin{array}{l}L / \delta=2500 \\
m=10260 \\
I=0.249470\end{array}$ & B54 & $\begin{array}{l}L / \delta=2575 \\
m=7058 \\
I=0.227698\end{array}$ \\
\hline B 15 & $\begin{array}{l}L / \delta=1050 \\
m=19128 \\
I=0.137816\end{array}$ & $\mathrm{~B} 25$ & $\begin{array}{l}L / \delta=1750 \\
m=19888 \\
I=0.234414\end{array}$ & B35 & $\begin{array}{l}L / \delta=2600 \\
m=19939 \\
I=0.348748\end{array}$ & B45 & $\begin{array}{l}L / \delta=3000 \\
m=13909 \\
I=0.338211\end{array}$ & B55 & $\begin{array}{l}L / \delta=3000 \\
m=8796 \\
I=0.283784\end{array}$ \\
\hline
\end{tabular}

Natural frequencies and mechanical properties of the 25 bridges analysed in the parametric study

Masses per unit length are given in kilograms per meter. Moments of inertia are given in $\mathrm{m}^{4}$. 


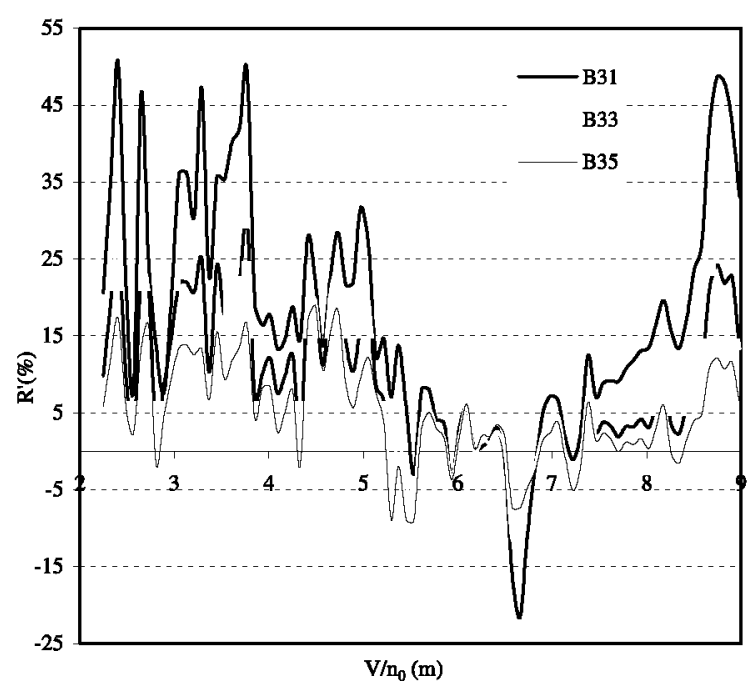

Fig. 12. Reduction of the maximum accelerations for bridges $\mathrm{B} 31, \mathrm{~B} 33$ and B35

where $R_{\text {ref }}(\lambda)$ and $R_{\text {ref }}^{\prime}(\lambda)$ are the reductions for bridge $\mathrm{B} 11$ and $\gamma\left(n_{0}, I\right), \gamma^{\prime}\left(n_{0}, I\right)$ are the intensities of reduction for a bridge with natural frequency $n_{0}$ and moment of inertia $I . R_{\text {ref }}(\lambda)$ and $R_{\text {ref }}^{\prime}(\lambda)$ are shown in Figs. 13 and 14 as a function of the wavelength. The values of the intensities of reduction are shown in Table 2 for the 25 bridges.

Substituting the values given by Eqs. (8a) and (8b) in Eqs. (7a) and (7b) an approximation to the impact coefficient $\Phi_{\mathrm{i}}$ can be obtained as follows:

$\Phi_{\mathrm{i}, \mathrm{app}}\left(\lambda, n_{0}, I\right)=\Phi_{\mathrm{c}}(\lambda)\left[1-\gamma\left(n_{0}, I\right) \frac{R_{\mathrm{ref}}(\lambda)}{100}\right]$

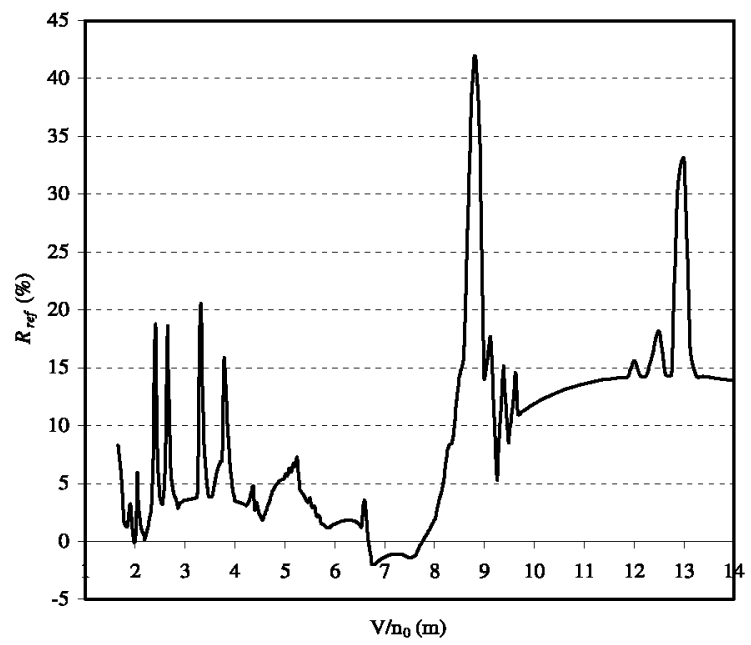

Fig. 13. Reduction of the impact coefficient for bridge B11 $\left(R_{\text {ref }}\right)$.

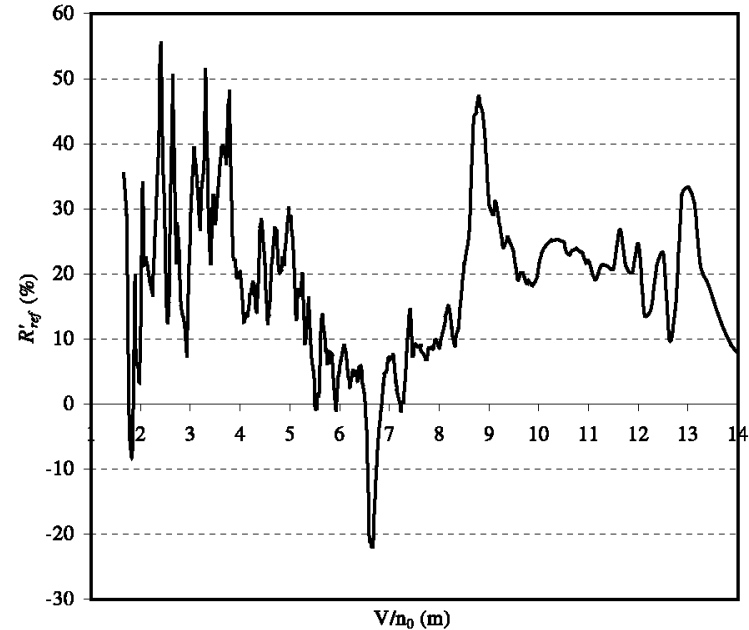

Fig. 14. Reduction of the maximum accelerations for bridge B11 $\left(R_{\text {ref }}^{\prime}\right)$.

Proceeding in an analogous way, an approximated value of the acceleration can be found, but in this case the first factor depends on the frequency and inertia of the bridge:

$a_{\mathrm{i}, \mathrm{app}}\left(\lambda, n_{0}, I\right)=a_{\mathrm{c}}\left(\lambda, n_{0}, I\right)\left[1-\gamma^{\prime}\left(n_{0}, I\right) \frac{R_{\mathrm{ref}}^{\prime}(\lambda)}{100}\right]$

Nevertheless, Eq. (10) can be modified taking into account the similarity formula (2b) to give

$a_{\mathrm{i}, \mathrm{app}}\left(\lambda, n_{0}, I\right)=a_{\mathrm{c}, \mathrm{ref}}(\lambda) \frac{m_{\mathrm{ref}}}{m}\left[1-\gamma^{\prime}\left(n_{0}, I\right) \frac{R_{\mathrm{ref}}^{\prime}(\lambda)}{100}\right]$

where $m_{\text {ref }}$ is the mass per unit length of bridge $\mathrm{B} 11, m$ is the mass per unit length of the bridge with frequency $n_{0}$ and moment of inertia $I ; a_{\mathrm{c}, \text { ref }}(\lambda)$ is the maximum acceleration of bridge B11 obtained from the moving loads model.

In Eqs. (9) and (11), approximations for the impact coefficient and maximum acceleration are given in such a way that the dependence on the wavelength is concentrated on $\Phi_{\mathrm{c}}(\lambda), a_{\mathrm{c}, \mathrm{ref}}(\lambda), R_{\mathrm{ref}}(\lambda)$ and $R_{\mathrm{ref}}^{\prime}(\lambda)$. The values of these factors are the same for all bridges of length $L=10 \mathrm{~m}$ and damping ratio $\zeta=1 \%$. As stated before, damping ratio is usually higher for short bridges (ERRI recommends using $1.7 \%$ for $10 \mathrm{~m} \mathrm{RC}$ or steel bridges [9]); however, bridge response is characterized by sharper peaks if lighter damping is assumed, and therefore $1 \%$ damping has been selected as these puts a tougher test for the approximated method presented herein.

Eqs. (9) and (11) take into account the train-bridge interaction and are easy to apply: $\Phi_{\mathrm{c}}(\lambda)$ and $a_{\mathrm{c}, \mathrm{ref}}(\lambda)$ can be computed using the moving loads model; $R_{\text {ref }}(\lambda)$ and $R_{\text {ref }}^{\prime}(\lambda)$ are shown in Figs. 13 and 14; finally, the intensities of reduction can be obtained from Table 2 . The 
Table 2

Intensities of reduction for the impact coefficients and maximum accelerations

\begin{tabular}{|c|c|c|c|c|c|c|c|c|c|}
\hline \multicolumn{2}{|c|}{$n_{01}=8 \mathrm{~Hz}$} & \multicolumn{2}{|c|}{$n_{02}=10.23 \mathrm{~Hz}$} & \multicolumn{2}{|c|}{$n_{03}=12.46 \mathrm{~Hz}$} & \multicolumn{2}{|c|}{$n_{04}=14.7 \mathrm{~Hz}$} & \multicolumn{2}{|c|}{$n_{05}=16.93 \mathrm{~Hz}$} \\
\hline B11 & $\begin{array}{l}y=1.0 \\
y^{\prime}=1.0\end{array}$ & $\mathrm{~B} 21$ & $\begin{array}{l}\gamma=1.33 \\
\gamma^{\prime}=1.20\end{array}$ & B31 & $\begin{array}{l}\gamma=1.17 \\
\gamma^{\prime}=1.03\end{array}$ & B41 & $\begin{array}{l}y=0.97 \\
y^{\prime}=0.86\end{array}$ & B51 & $\begin{array}{l}\gamma=0.87 \\
\gamma^{\prime}=0.74\end{array}$ \\
\hline B12 & $\begin{array}{l}\gamma=0.74 \\
\gamma^{\prime}=0.80\end{array}$ & $\mathrm{~B} 22$ & $\begin{array}{l}\gamma=0.80 \\
\gamma^{\prime}=0.80\end{array}$ & B32 & $\begin{array}{l}\gamma=0,67 \\
\gamma^{\prime}=0.67\end{array}$ & $\mathrm{~B} 42$ & $\begin{array}{l}\gamma=0.63 \\
\gamma^{\prime}=0.6\end{array}$ & B52 & $\begin{array}{l}\gamma=0,63 \\
\gamma^{\prime}=0.58\end{array}$ \\
\hline B13 & $\begin{array}{l}\gamma=0.57 \\
\gamma^{\prime}=0.65\end{array}$ & $\mathrm{~B} 23$ & $\begin{array}{l}\gamma=0.53 \\
\gamma^{\prime}=0.57\end{array}$ & B33 & $\begin{array}{l}\gamma=0.44 \\
\gamma^{\prime}=0.47\end{array}$ & B43 & $\begin{array}{l}\gamma=0.44 \\
\gamma^{\prime}=0.45\end{array}$ & B53 & $\begin{array}{l}\gamma=0.49 \\
\gamma^{\prime}=0.47\end{array}$ \\
\hline B14 & $\begin{array}{l}y=0.44 \\
y^{\prime}=0.52\end{array}$ & B24 & $\begin{array}{l}\gamma=0.37 \\
\gamma^{\prime}=0.42\end{array}$ & B34 & $\begin{array}{l}\gamma=0.31 \\
\gamma^{\prime}=0.34\end{array}$ & B44 & $\begin{array}{l}\gamma=0.33 \\
y^{\prime}=0.35\end{array}$ & B54 & $\begin{array}{l}\gamma=0.39 \\
\gamma^{\prime}=0.39\end{array}$ \\
\hline B15 & $\begin{array}{l}y=0.34 \\
y^{\prime}=0.43\end{array}$ & $\mathrm{~B} 25$ & $\begin{array}{l}\gamma=0.26 \\
\gamma^{\prime}=0.31\end{array}$ & B35 & $\begin{array}{l}\gamma=0.21 \\
\gamma^{\prime}=0.25\end{array}$ & B45 & $\begin{array}{l}y=0.25 \\
y^{\prime}=0.27\end{array}$ & B55 & $\begin{array}{l}\gamma=0.33 \\
\gamma^{\prime}=0.34\end{array}$ \\
\hline
\end{tabular}

values of $\gamma\left(n_{0}, I\right)$ and $\gamma^{\prime}\left(n_{0}, I\right)$ corresponding to bridges different from the ones analysed in this study can be computed by linear interpolation.

\subsection{Approximate formulas for the intensities of reduction}

A final improvement is presented in this section that simplifies the computation of the impact coefficient and maximum acceleration for any bridge of $10 \mathrm{~m}$ of span length and damping ratio $\zeta=1 \%$.

If the values of the intensities of reduction shown in Table 2 are represented as a function of the moment of inertia using logarithmic scales, Figs. 15 and 16 are obtained. As can be seen, the values of $\gamma$ and $\gamma^{\prime}$ lie on nearly straight lines, each of them corresponding to a different value of the fundamental frequency.

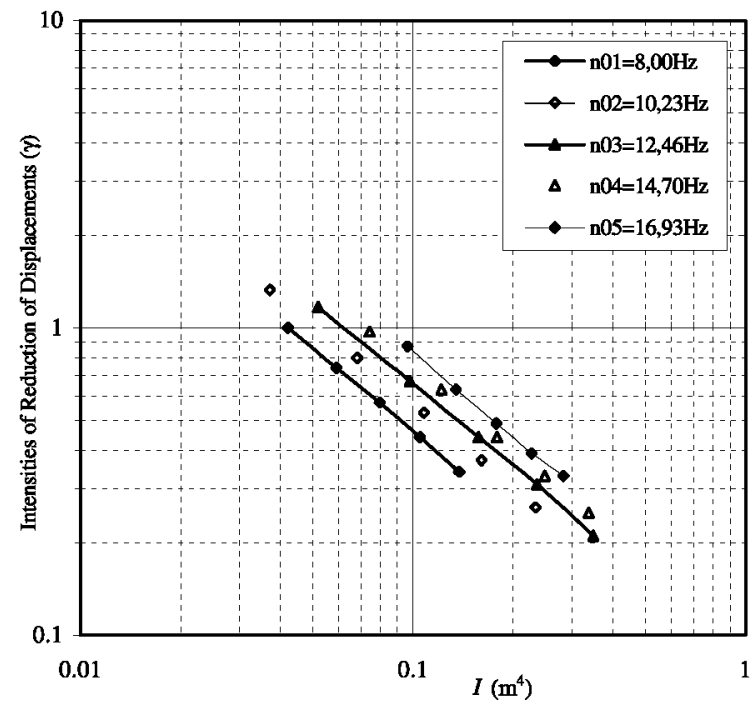

Fig. 15. Intensities of reduction for the impact coefficients $(\gamma)$.

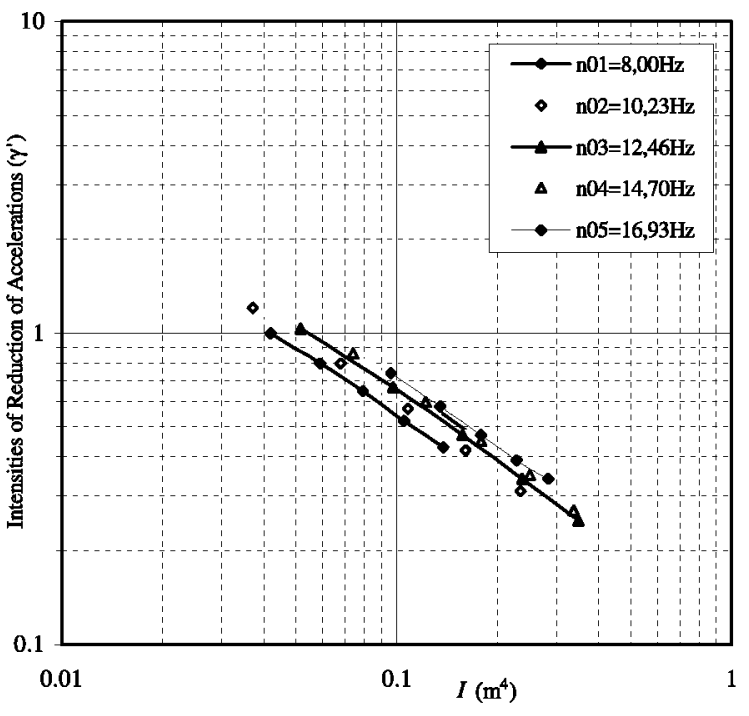

Fig. 16. Intensities of reduction for the maximum accelerations $\left(\gamma^{\prime}\right)$.

This suggests that the intensities can be approximated by expressions such as

$\gamma\left(n_{0}, I\right)=K\left(n_{0}\right) I^{N}$

$\gamma^{\prime}\left(n_{0}, I\right)=K^{\prime}\left(n_{0}\right) I^{N^{\prime}}$

The exponents $N=-0.93$ and $N^{\prime}=-0.74$ produce the best fitting for the straight lines in Figs. 15 and 16. In addition, if the values of the coefficients $K\left(n_{0}\right)$ and $K^{\prime}\left(n_{0}\right)$ are investigated, they are found to be almost linearly dependent on the value of the frequency. Straightforward calculations allow expressing them as

$K\left(n_{0}\right)=0.0143+0.00498 n_{0}$

$K^{\prime}\left(n_{0}\right)=0.0715+0.00353 n_{0}$ 
If these values are substituted into Eqs. (12a) and (12b), two approximate expressions for the intensities of reduction are obtained:

$\gamma\left(n_{0}, I\right)=\left(0.0143+0.00498 n_{0}\right) I^{-0.93}$

$\gamma^{\prime}\left(n_{0}, I\right)=\left(0.0715+0.00353 \cdot n_{0}\right) I^{-0.74}$

where the natural frequency is to be expressed in $\mathrm{Hz}$, and the moment of inertia in $\mathrm{m}^{4}$.

Using Eqs. (14a) and (14b) in Eqs. (9) and (11) produces approximate values of the impact coefficients and maximum accelerations that are in very good agreement

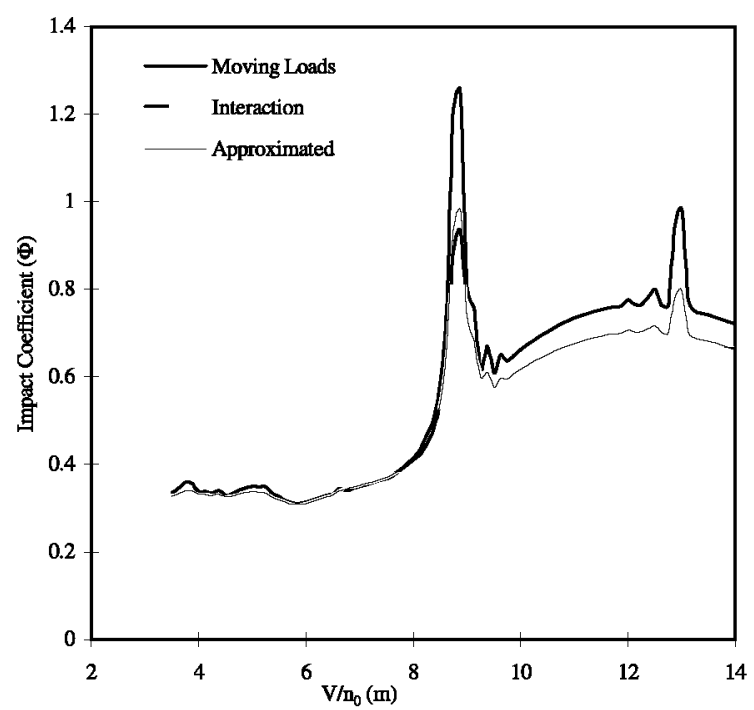

Fig. 17. Impact coefficients for bridge B13.

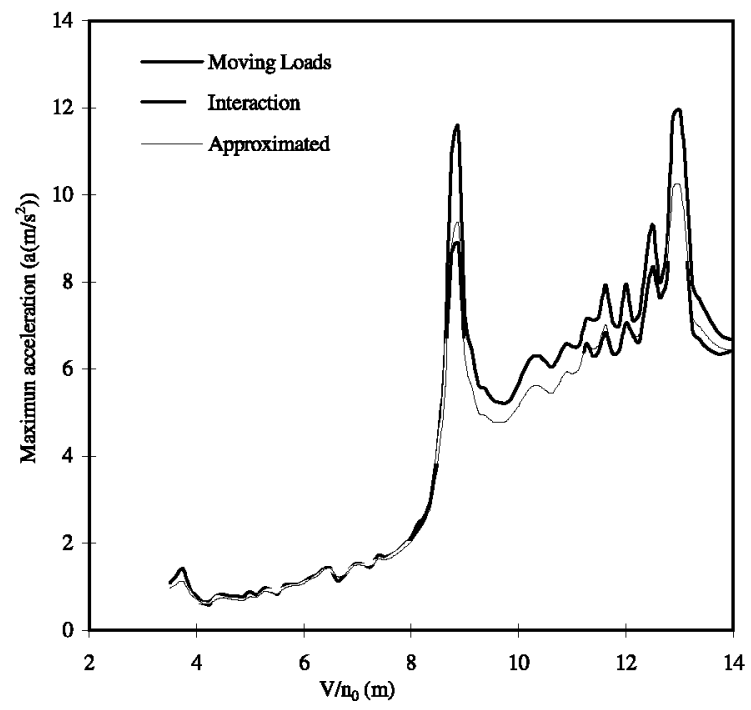

Fig. 18. Maximum accelerations for bridge B15. with those obtained from the train-bridge interaction model. The comparison is highly satisfactory for all the 25 bridges analysed in the parametric study: in only four of them the peak values are underestimated by $<10 \%$, while in the rest the approximated values are slightly greater than the exact ones, or in many cases they are almost identical.

Figs. 17-22 show the comparison between the approximated values and the ones obtained from the interaction model. The impact coefficients and maximum accelerations computed with the moving loads model are

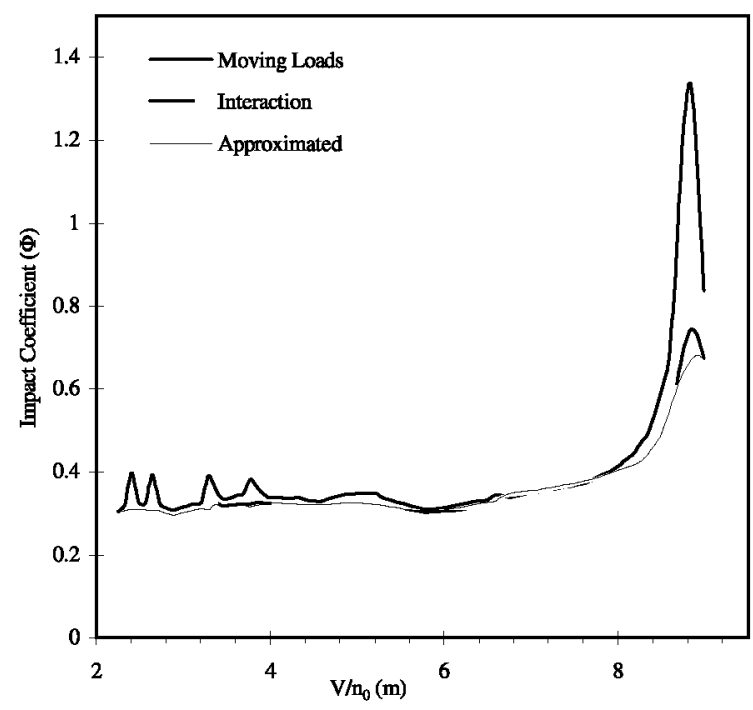

Fig. 19. Impact coefficients for bridge B31.

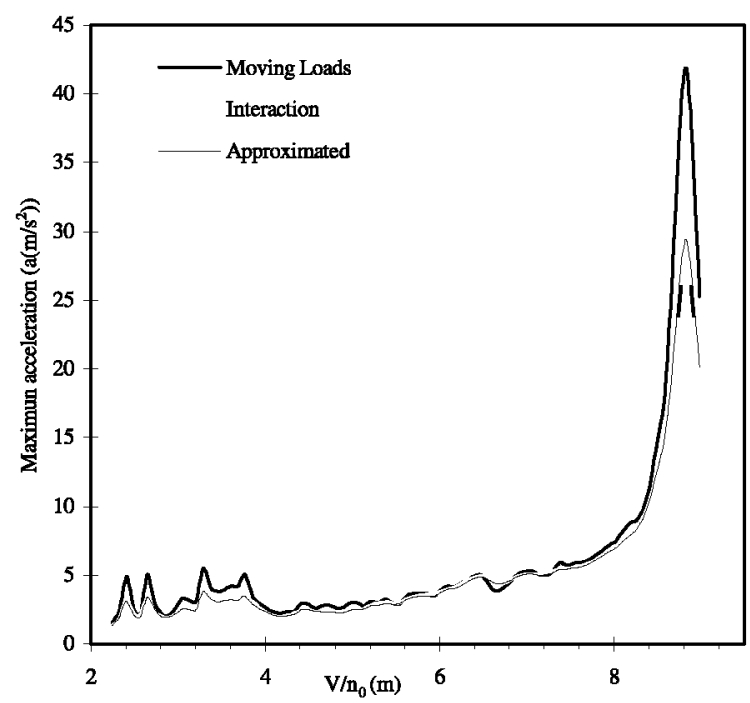

Fig. 20. Maximum accelerations for bridge B32. 


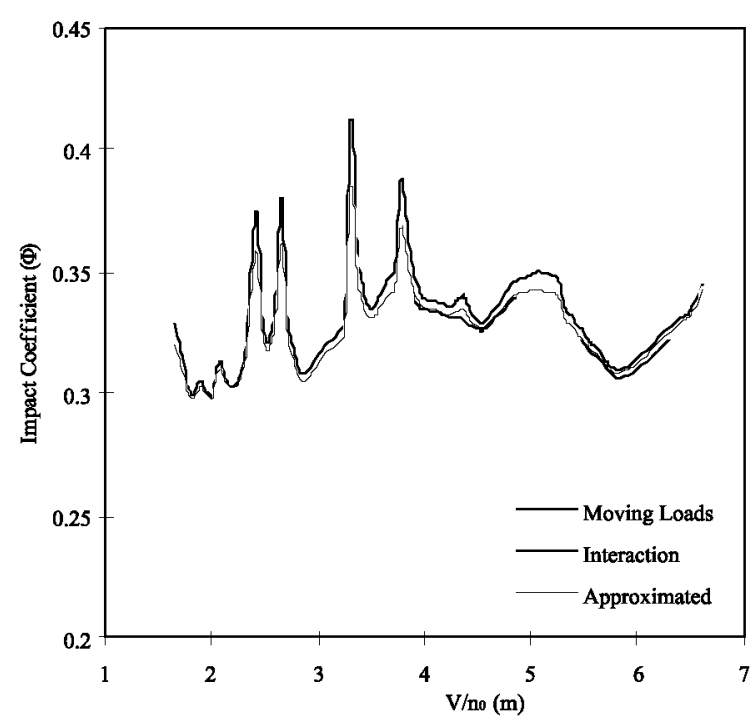

Fig. 21. Impact coefficients for bridge B55.

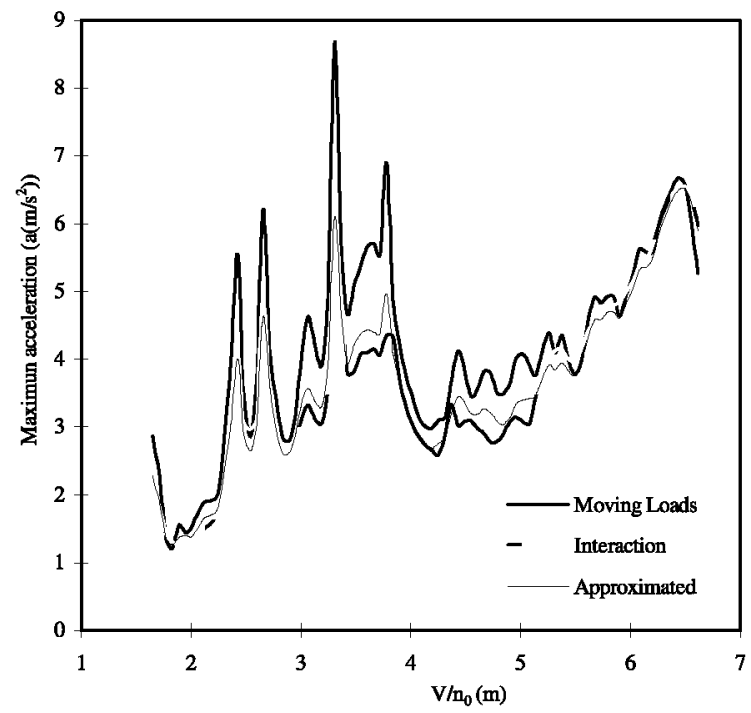

Fig. 22. Maximum accelerations for bridge B52.

also included in order to give an idea of the degree of precision of the approximation proposed in this section.

\section{Conclusions}

The effects of the load distribution through the sleepers and ballast layer have been analysed. It has been shown that the maximum accelerations of the deck are not significantly affected by this factor except for the shorter values of the wavelength $\left(\lambda=V / n_{0} \leqslant 4-5 \mathrm{~m}\right)$. The calculations have been performed considering that each axle load is distributed uniformly over a length of $1 \mathrm{~m}$.

The train-bridge interaction causes reductions of considerable importance in the maximum displacements and accelerations of short bridges. The effects of the ICE-2 train on 25 simply supported bridges of $10 \mathrm{~m}$ of span length have been investigated, and it has been found that the reductions obtained in bridges with different natural frequency and moment of inertia are nearly proportional to each other. The coefficients of proportionality, called intensities of reduction, have been computed for displacements and accelerations.

The intensities of reduction can be very accurately approximated using numerical expressions. The comparison of the impact coefficients and maximum accelerations obtained from the interaction model with those obtained from the approximated method proposed herein is highly satisfactory. Nevertheless, further study is required in order to prove the validity of the procedure for different trains and span lengths; investigation on this subject is being carried out at the moment and the results will be presented in future publications.

\section{References}

[1] Alvarez L, Díaz J, Fernández J, Ley J, Santos J, Santos F. Numerical simulation model for railway bridges under high speed traffic. Cuadernos Intemac 1996;24.

[2] Biggs JM. Introduction to structural dynamics. New York: McGraw-Hill; 1964.

[3] Bleich F. Theorie und berechnung der eisernen brücken. Springer-Verlag; 1924.

[4] Bresse. Cours de mecanique. Paris: Ecole de Ponts et Chaussées; 1880.

[5] Commité Européen de Normalisation (CEN). Eurocode 1-basis of design and actions on structures. CEN, 1995.

[6] ERRI D-214 Committee. Numerical investigation of the effect of track irregularities at bridge resonance: draft Report 5. Utrecht: European Rail Research Institute (ERRI); 1999.

[7] ERRI D-214 Committee. Ponts rails pour vitesses $>200$ $\mathrm{km} / \mathrm{h}$ et $<350 \mathrm{~km} / \mathrm{h}$. Conditions de déformation dynamiques. Rapport interimaire. Utrecht: European Rail Research Institute (ERRI); 1997.

[8] ERRI D-214 Committee. Ponts rails pour vitesses $>200$ $\mathrm{km} / \mathrm{h}$. Interaction train-pont. Rapport d'information. European Rail Research Institute (ERRI); 1997.

[9] ERRI D-214 Committee. Ponts rails pour vitesses $>200$ $\mathrm{km} / \mathrm{h}$. Rapport Final. European Rail Research Institute (ERRI); 2000.

[10] Frýba L. Vibration of solids and structures under moving loads. Groningen: Noordhoff; 1972.

[11] Frýba L. Dynamics of railway bridges. London: Thomas Telford; 1996.

[12] Frýba L. A rough assessment of railway bridges for high speed trains. Eng Struct 2001;23(5):548-56. 
[13] Inglis CE. A mathematical teatrise on vibrations in railway bridges. Cambridge: Cambridge University Press; 1934.

[14] Mancel F. Cedypia: analytical software for calculating dynamic effects on railway bridges. In: Proceedings of the 4th Conference of the European Association for Structural Dynamics. The Netherlands: A.A. Balkema; 1999.

[15] Ministerio de Obras Públicas y Urbanismo (MOPU). Instrucción relativa a las acciones a considerar en el proyecto de puentes de ferrocarril, MOPU, in preparation.

[16] Museros P, Vivero G, Alarcón E. Moving loads in railway bridges: The Spanish Code Approach. In: Proceedings of the 4th Conference of the European Association for Structural Dynamics. The Netherlands: A.A. Balkema; 1999.

[17] Nasarre J. Algunas consideraciones sobre la necesidad de cálculos dinámicos de los puentes ferroviarios para velocidades elevadas. In: Actas. 1. Primer Congreso de la
Asociación Científico Técnica del Hormigón Estructural (ACHE), Seville, Spain, 1999.

[18] Olsson M. On the fundamental moving load problem. J Sound Vibr 1991;145(2):299-307.

[19] Stokes GG, Mathematical and physical papers, Cambridge, 1847.

[20] Swanson Analysis System Inc. Ansys V5.0: Theory Manual. Houston: Swanson Analysis System Inc; 1992.

[21] Timoshenko SP, Young DH. Vibration problems in engineering. third ed. New York: D. Van Nostrand; 1955.

[22] Willis R. Appendix to the report of the commissions appointed to inquire into the application of iron to railway structures. London: H. M. Stationery office; 1849.

[23] Yang YB, Yau JD. Vehicle-bridge interaction element for dynamic analysis. J Struct Eng (ASCE) 1997;123(11): 1512-8. 